\title{
Nonlinear scalar coupling models and proton-nucleus scattering observables
}

\author{
A. Eiras and G. Krein \\ Instituto de Física Teórica, Universidade Estadual Paulista, \\ Rua Pamplona, 145, 01405-900 São Paulo, Brazil
}

(Received 8 November 1993)

\begin{abstract}
The effects of nonlinear scalar field couplings on elastic proton-nucleus scattering observables are investigated using a relativistic impulse approximation. Nonlinear couplings affect in a nontrivial way the effective nucleon mass and the nuclear scalar and vector densities. Modifications on the densities might have observable consequences on scattering observables. Our investigation indicates that the description of the observables for the reactions $p-{ }^{16} \mathrm{O}$ and $p-{ }^{40} \mathrm{Ca}$ at $200 \mathrm{MeV}$ are not greatly modified with the use of nonlinear models in comparison with the description using linear models.
\end{abstract}

PACS number(s): 25.40.Cm, 24.10.Jv, 21.65. $+\mathrm{f}$

Nonrelativistic nuclear models have been used with success in the last 50 years to describe the ground- and excited-state properties of nuclei. Nevertheless, in the near future nuclear systems will be probed at extreme conditions of density and temperature and obviously nonrelativistic models are inadequate to describe these phenomena. This has motivated the development of relativistic models for the nucleus based on the methods of relativistic quantum field theory and an extensive literature on this subject has been accumulated in recent years.

Calculations based on the original Walecka [1] meanfield model have achieved a reasonably good description of the properties of nuclear ground states and have provided a remarkably good description of elastic nucleonnucleus scattering observables at intermediate energies. In spite of the successes, there are serious problems related to the vacuum structure of the models. In particular, the vacuum polarization effects of the effective scalar field have undesirable features. In Walecka-type models, the effective nucleon mass in nuclear matter is of the order of $50 \%$ of its free-space value (renormalization can reduce the effect to $70 \%$ ). This low effective nucleon mass has the effect of producing among other things nucleon-antinucleon instabilities. Although there have been attempts to construct consistent field theoretic models for the nucleus $[2,3]$, much still remains to be done. The main difficulty is that one is dealing with complicated nonperturbative effects in field theory, and there has been no systematic approximation scheme which is appropriate for dealing with such effects. With the purpose of including vacuum polarization effects in an effective way in a mean-field model, Zimanyi and Moszkowski [4] introduced an effective Lagrangian with a derivative coupling. The main effect is to reduce the intensity of the scalar field coupling to the fermions. This nonlinear model introduces no additional parameters in comparison to the standard two-parameter relativistic mean-field model, although the functional form of the coupling is, in some sense, arbitrary.

Nonlinear scalar coupling models have been employed to study the properties of nuclear matter at finite temper- ature [5], the real part of the optical potential [6], and the properties of finite nuclei [7]. One important consequence of the nonlinear coupling is the modification of the behavior of the effective mass as function of the density. For finite nuclei, the value of the effective mass at saturation plays an important role in all the properties studied [7]. The vector and scalar densities predicted from different types of scalar couplings differ in the central region of the nucleus. For the surface region they give similar results. These differences are more important for the scalar density, since this quantity is more sensitive to the lower component of the Dirac spinor than the vector density. This is an important result which might have observable consequences. The details of the structure of the nucleus affect in a nontrivial way the nucleon-nucleus scattering observables. This point was made clear by Murdock and Horowitz [8] in a recent study. These authors effectuated a relativistic microscopic calculation of the spin observables of elastic proton scattering from several nuclei at laboratory kinetic energies near $200 \mathrm{MeV}$. In particular, their calculations show that for densities obtained from a relativistic Hartree approximation, the fits to the scattering observables are poorer for c.m. angles larger than $50^{\circ}$. They point out that vacuum fluctuations might have significant effects on the densities and this may be the cause for the discrepancies. It is therefore interesting to study the consequences of the different couplings on the scattering observables. It is the purpose of the present work to perform such a study.

The relevant nuclear structure input to the relativistic impulse approximation are the nuclear densities

TABLE I. The functionals $m^{*}(\sigma)$ and $g_{S}(\sigma)$.

\begin{tabular}{lcc}
\hline & $m^{*}(\sigma)$ & $g_{S}(\sigma)=-M d m^{*} / d \sigma$ \\
\hline (a) & $1-g_{S} \sigma / M$ & $g_{S}$ \\
(b) & $1-\tanh \left(g_{S} \sigma / M\right)$ & $g_{S} m^{*}(\sigma)\left[2-m^{*}(\sigma)\right]$ \\
(c) & $\left(1+g_{S} \sigma / M\right)^{-1}$ & $g_{S}\left(m^{*}(\sigma)\right)^{2}$ \\
\hline \hline
\end{tabular}



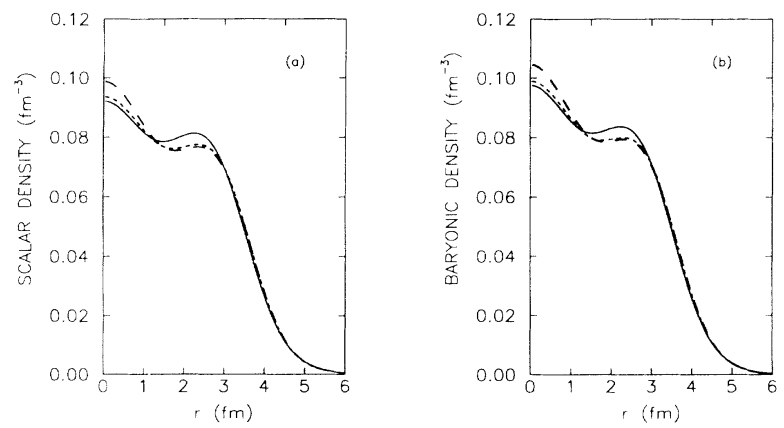

FIG. 1. Comparison of scalar (a) and baryon (b) densities calculated for different $m^{*}(\sigma)$ (Table I). Solid curves stand for case (a), small-dashed curves refer to case (b), and long-dashed ones stand for case (c).

$$
\begin{aligned}
\rho_{S}(r) & =\sum_{\alpha}^{A} \bar{\phi}_{\alpha}(r) \phi_{\alpha}(r), \\
\rho_{V}(r) & =\sum_{\alpha}^{A} \phi_{\alpha}^{\dagger}(r) \phi_{\alpha}(r), \\
\rho_{3}(r) & =\sum_{\alpha_{p}}^{Z} \phi_{\alpha_{p}}^{\dagger}(r) \phi_{\alpha_{p}}(r)-\sum_{\alpha_{n}}^{N} \phi_{\alpha_{n}}^{\dagger}(r) \phi_{\alpha_{n}}(r), \\
\rho_{c}(r) & =\sum_{\alpha_{p}}^{Z} \phi_{\alpha_{p}}^{\dagger}(r) \phi_{\alpha_{p}}(r), \\
\hat{\mathbf{r}}^{i} \rho_{T}(r) & =\sum_{\alpha}^{A} \bar{\phi}_{\alpha}(r) \sigma^{0 i} \phi_{\alpha}(r) .
\end{aligned}
$$

The $\phi_{\alpha}$ are single-particle Dirac spinors ( $p$ and $n$ indicate proton and neutron), solutions of the Dirac equation

$$
\left\{-i \boldsymbol{\alpha} \cdot \boldsymbol{\nabla}+\beta\left[M+U_{S}(r)\right]+U_{V}(r)\right\} \phi_{\alpha}(\mathbf{r})=\epsilon_{\alpha} \phi_{\alpha}(\mathbf{r}),
$$

where $U_{S}(r)$ depends on the mean scalar field $\sigma$

$$
U_{S}(r)=M\left[m^{*}(\sigma(r))-1\right],
$$

and $U_{V}(r)$ depends on the mean fields corresponding to the $\omega$ and $\rho$ mesons and on the photon field $A$,

$$
U_{V}(r)=g_{\omega} \omega_{0}(r)+\frac{1}{2} g_{\rho} \tau_{3} \rho_{0}(r)+\frac{1}{2} e\left(1+\tau_{3}\right) A_{0}(r) .
$$

In Eq. (3), $g_{S}(\sigma)$ is given by

$$
g_{S}(\sigma)=-M \frac{d m^{*}(\sigma)}{d \sigma},
$$

where $m^{*}(\sigma)$ is a functional of the scalar field $\sigma$ which comes from a term in the the Lagrangian density of the form

$$
\mathcal{L}_{\sigma N}=M m^{*}(\sigma) \bar{\psi} \psi
$$

Note that the only difference, compared to the usual linear models, appears in the equation for the $\sigma$ field; instead of linear, it is now nonlinear. The solution of this equation can be written formally as an integral equation

$$
\sigma(r)=\int_{0}^{\infty} d r^{\prime} r^{\prime 2} G_{\sigma}\left(r, r^{\prime}\right)\left[-g_{S}\left(\sigma\left(r^{\prime}\right)\right) \rho_{S}\left(r^{\prime}\right)\right],
$$

where $G_{\sigma}\left(r, r^{\prime}\right)$ is the Green's function of the operator $\nabla^{2}-m_{\sigma}^{2}$.

The mass functionals $m^{*}(\sigma)$ and the corresponding coupling functionals $g_{S}(\sigma)$ which we use in this work are shown in Table I. Those are the same ones used in past works, Refs. [6,7].

The constraints on the form of $m^{*}(\sigma)$ are that (a) at zero density $M m^{*}(\sigma) \longrightarrow M$ [see Eq. (6)], and (b) at higher densities, the effective nucleon mass never goes negative and approaches zero asymptotically. Clearly, case (a) in Table I, which corresponds to the usual linear coupling model, does not satisfy the last constraint. We consider this case to compare with the nonlinear couplings in (b) and (c). Case (b) has proved to be best suited to describe the energy dependence of the real part of the optical potential for energies up to $400 \mathrm{MeV}$ [6]. Finally, case (c) is the Zimanyi-Moszkowski nonlinear model [4].

We consider proton scattering from spin-zero targets. The relevant scattering observables are the differential cross section $d \sigma / d \Omega$, the analyzing power $A_{y}(\theta)$, and the spin rotation $Q(\theta)$, where $\theta$ is the c.m. angle. These will be determined by employing the relativistic impulse approximation (RIA) developed by Murdock and Horowitz [8]. Their approach is an extension of the original RIA
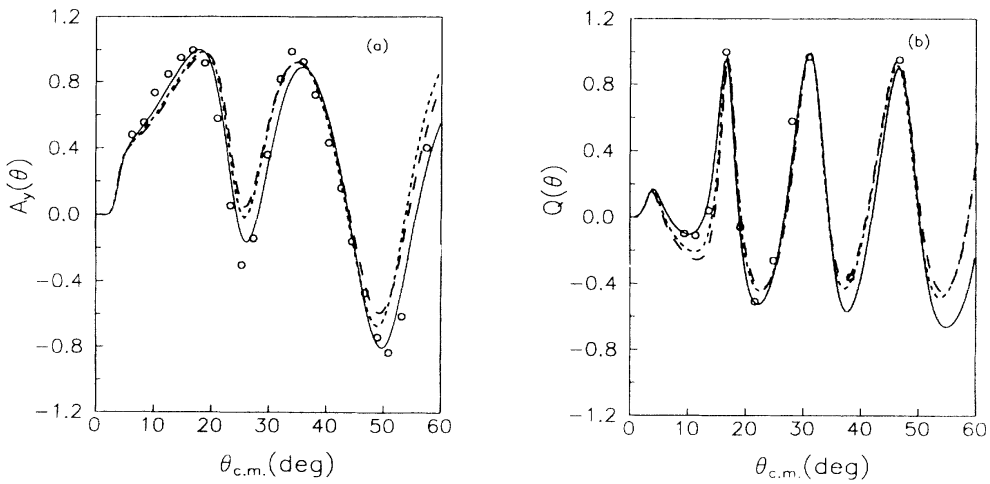

FIG. 2. Analyzing power and spin rotation for $p-{ }^{16} \mathrm{O}$ at laboratory kinetic energy $E=200 \mathrm{MeV}$ for different $m^{*}(\sigma)$ (Table I). Solid curves stand for case (a), small-dashed curves refer to case (b), and long-dashed ones stand for case (c). For experimental data, see Ref. [8]. 


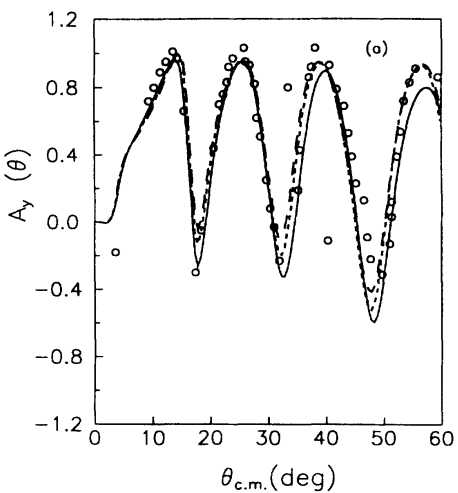

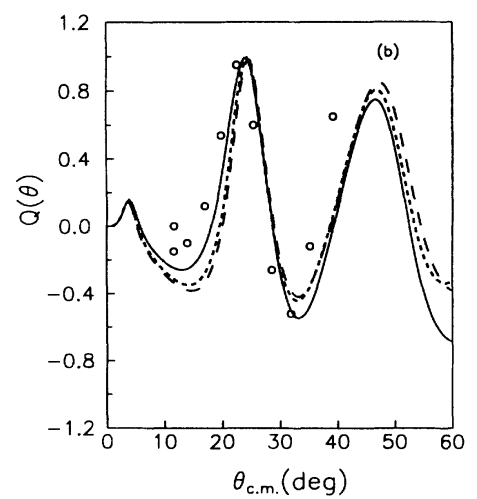

FIG. 3. Same as in Fig. 2 for $p^{40} \mathrm{Ca}$. of McNeil, Ray, and Wallace [9] in several important aspects: exchange effects in the optical potential are included, ambiguities with respect to the pion coupling in the relativistic $N N$ amplitudes are resolved by using pseudovector coupling instead of pseudoscalar coupling, and medium modifications from Pauli blocking are incorporated using relativistic Brueckner results for nuclear matter in a local density approximation. Here we do not go into the details of the formalism, we simply refer the reader to the original publications in Refs. $[8,10]$ for details.

The coupled set of Dirac and Klein-Gordon equations is solved by iteration, following the strategy of Ref. [11]. We start solving the Dirac equation with Woods-Saxon forms for the $U_{S}$ and $U_{V}$. With the solutions of the Dirac equation we obtain the densities $\rho_{S}, \rho_{V}, \rho_{3}$, and $\rho_{c}$. These densities are used to solve the Klein-Gordon equations for the meson fields. The integral equation for the $\sigma$ field, Eq. (7), is solved by iteration. The meson fields are then used to get the potentials $U_{S}$ and $U_{V}$, Eqs. (3) and (4), and these are used to solve the Dirac equation. The process is iterated to convergence.

Figures 1(a) and 1(b) show, respectively, the scalar and baryonic densities for ${ }^{40} \mathrm{Ca}$ for different $m^{*}(\sigma)$ of Table I. As remarked earlier, the nonlinear terms introduce a more significant influence in the central region of the nucleus, where the deviation from the linear scalar coupling (solid lines) is not appreciable. There are no significant differences among the various couplings at the surface of the nucleus. Similar features were observed for different nuclei [7].

With respect to the scattering observables, we found a relative insensitivity on the functional form of $m^{*}(\sigma)$. Figures 2 and 3 illustrate the results for the reactions $p-{ }^{16} \mathrm{O}$ and $p-{ }^{40} \mathrm{Ca}$ at laboratory kinetic energy $E=200$ $\mathrm{MeV}$. For small scattering angles, the agreement with the data is perfect, with no significant difference among the linear and nonlinear couplings. The main contribution of the nonlinear terms appears for scattering angles larger than $25^{\circ}$; the effect is larger for the reactions higher mass target nuclei.

We have also studied the observables at higher and lower energies and on different target nuclei $\left({ }^{12} \mathrm{C},{ }^{48} \mathrm{Ca}\right.$, ${ }^{90} \mathrm{Zr}$, and ${ }^{208} \mathrm{~Pb}$ ), and have also used an alternative form for the functional $m^{*}(\sigma)$, the exponential form $m^{*}(\sigma)=$ $\exp \left(-g_{S} \sigma / m\right)$. The results are essentially the same as described above: independence on the functional form of $M^{*}(\sigma)$ and the effect of the nonlinearity is larger for scattering angles larger than $25^{\circ}$.

On the basis of the results of this study, we conclude that the modifications on the target scalar and vector densities due to the nonlinear scalar couplings introduce small deviations on the scattering observables in comparison to the results obtained using the usual linear scalar coupling of the Walecka model. The deviations from the linear coupling are larger for angles larger than $25^{\circ}$, and the effect is larger for the reactions on higher mass nuclei. An important point of the study is that the results for the scattering observables are relatively insensitive to the different functional forms for the scalar coupling. A relative insensitivity is observed also on the differential cross sections, in both cases, $p-{ }^{16} \mathrm{O}$ and $p-{ }^{40} \mathrm{Ca}$. Finally, it appears that the important role of the nonlinear couplings is to provide a more reasonable value for the effective nucleon mass, and a consequent lower nuclear compressibility. Once this is achieved, the predictions of the models are relatively insensitive to the particular functional form used for $m^{*}(\sigma)$.

A.E. was supported and G.K. was partially supported by CNPq (Brazil).
[1] J. D. Walecka, Ann. Phys. (N.Y.) 83, 491 (1974).

[2] M. P. Allendes and B. D. Serot, Phys. Rev. C 45, 2975 (1992).

[3] G. Krein, M. Nielsen, R. D. Puff, and L. Wilets, Phys. Rev. C 47, 2485 (1993); M. E. Bracco, A. Eiras, G. Krein, and L. Wilets, Phys. Rev. C 49, 1299 (1994).

[4] J. Zimanyi and S. A. Moszkowski, Phys. Rev. C 42, 1416
(1990).

[5] Zhi-Xin Qian, Hong-Qiu Song, and Ru-Kung Su, Phys. Rev. C 48, 154 (1993).

[6] H. Feldmeier and J. Lindner, Z. Phys. 341, 83 (1991).

[7] W. Koepf, M. M. Sharma, and P. Ring, Nucl. Phys. A533, 95 (1991).

[8] D. P. Murdock and C. J. Horowitz, Phys. Rev. C 35, 
$1442(1987)$.

[9] J. A. McNeil, J. R. Shepard, and S. J. Wallace, Phys. Rev. Lett. 50, 1439 (1983); J. R. Shepard, J. A. McNeil, and S. J. Wallace, ibid. 50, 1443 (1983); J. A. McNeil, J. R. Shepard, and S. J. Wallace, Phys. Rev. C 27, 2123
(1983).

[10] C. J. Horowitz, Phys. Rev. C 31, 1461 (1985).

[11] C. J. Horowitz and B. D. Serot, Nucl. Phys. A368, 508 (1981). 